\title{
BMSAP
}

Bulletins et mémoires de la Société d'Anthropologie de Paris

BMSAP

34 (1) | 2022

Entre vivants et morts : regards croisés sur une frontière relative et fluctuante

\section{De l'interprétation des liens entre vivants et morts dans l'espace habité maya Classique}

Interpreting ties between the living and the dead in Ancient Mayan living spaces

Hemmamuthé Goudiaby

\section{CpenEdition}

Journals

Édition électronique

URL : https://journals.openedition.org/bmsap/9378

DOI : $10.4000 /$ bmsap.9378

ISSN : 1777-5469

Éditeur

Société d'Anthropologie de Paris

Référence électronique

Hemmamuthé Goudiaby, « De l'interprétation des liens entre vivants et morts dans l'espace habité maya Classique », Bulletins et mémoires de la Société d'Anthropologie de Paris [En ligne], 34 (1) | 2022 mis en ligne le 10 février 2022, consulté le 13 février 2023. URL : http://journals.openedition.org/ bmsap/9378; DOI : https://doi.org/10.4000/bmsap.9378

\section{cc) (i) $\Theta$}

Creative Commons - Attribution - Pas d'Utilisation Commerciale - Pas de Modification 4.0 International - CC BY-NC-ND 4.0

https://creativecommons.org/licenses/by-nc-nd/4.0/ 


\title{
De l'interprétation des liens entre vivants et morts dans l'espace habité maya Classique
}

\author{
Interpreting ties between the living and the dead \\ in ancient mayan living spaces
}

Hemmamuthé Goudiaby (D) ${ }^{1 *}$

1 UMR 8096 ArchAm, Paris, France

* h.goudiaby@outlook.fr

Reçu : 10 mai 2021 ; accepté : 17 juillet 2021

Bulletins et Mémoires de la Société d'Anthropologie de Paris

Cette note fait suite à une communication présentée lors des $1846^{\text {es }}$ journées de la Société d'Anthropologie de Paris

dans le cadre de la session "Entre vivants et morts : regards croisés sur une frontière relative et fluctuante"

Résumé - Il n'est pas toujours possible de distinguer, au sein d'un ensemble funéraire apparemment homogène, l'existence de différences statutaires entre les défunts. Pourtant, lorsqu'elles existent, celles-ci définissent souvent la manière dont les vivants interagissent avec les morts : comment ils les inhument, combien de temps ils s'en souviennent, quel pouvoir ils leur attribuent. Comment identifier archéologiquement ces distinctions et, surtout, leur donner du sens ? Les pratiques funéraires mayas, étroitement liées aux habitats dans lesquels les morts sont inhumés, obéissent à une grammaire spatiale dont on perçoit aujourd'hui encore les échos parmi leurs descendants. En associant observations archéologiques issues de cas concrets et données ethnographiques, il est possible de rendre partiellement aux défunts leur place dans une hiérarchie funéraire plus nuancée que la figure parfois omniprésente des "ancêtres" ne le laisse envisager.

Mots clés - pratiques funéraires, Mayas, habitat, ancêtres

\begin{abstract}
When faced with homogeneous funerary complexes, it is not always possible to discern the existence of different statuses among the dead. When they do exist, such differences often define how the living interacted with their forebears: how they were buried, how long they were remembered, what powers were bestowed upon them. As archaeologists, how do we identify such differences and, above all, what do they mean? Ancient Mayan funerary practices were closely associated with the habitats in which the dead were buried and they obeyed a spatial codification that still echoes among their descendants to this day. By associating archaeological observations and ethnographical data, it becomes possible to partially restore the place of the dead in a funerary hierarchy of greater subtlety than the sometimes omnipresent figure of the "ancestor" tends to suggest.
\end{abstract}

Keywords - funerary practices, Maya, habitat, ancestors
Dans les sociétés modernes occidentales, le décès est souvent perçu comme un point dans le temps qui sépare nettement les états de vie et de mort. Il faut pourtant s'interroger : de quelle mort parle-t-on ? Car la mort biologique n'implique pas nécessairement la mort sociale ; de fait, c'est même rarement le cas tant qu'il reste des vivants pour interagir avec les défunts. Où tracer la limite à partir de laquelle un individu est considéré comme mort? Et si, parmi les morts, certains mouraient plus vite que d'autres ? Certains groupes humains ont poussé les interactions avec leurs défunts au point d'en faire un ciment social particulièrement puissant et indispensable, faisant en sorte de les retenir auprès d'eux le plus longtemps possible. La civilisation Maya en est un exemple, au moins durant son époque dite "Classique", laquelle s'étend de 250 à 1000 apr. J.-C. environ.

Durant cette période, la société maya s'organise en une multitude de cités indépendantes les unes des autres mais obéissant toutes plus ou moins au même modèle : un roi sacré, accompagné d'une cour de familles nobles, étend son influence sur un territoire aux limites floues et s'engage dans une série de jeux d'alliances et de rivalités politiques avec ses voisins. Ce système débouche en partie - d'autres facteurs n'étant pas à exclure - sur une situation intéressante : bien qu'elles partagent divers traits qui permettent bel et bien de parler d'une culture maya depuis le nord du Yucatan jusqu'à la côte Pacifique du Guatemala, chaque cité ou région n'en développe pas moins des variantes propres du thème de base. Ainsi, les Mayas inhument une partie de leurs morts directement sous le sol des habitats, ou parfois sous le sol des cours centrales de leurs résidences. Cependant, l'orientation et la position du corps, de même que le mobilier qui l'accompagne ou la localisation de la tombe, sont susceptibles de changer radicalement d'une cité à l'autre (Weiss-Krejci, 2006 ; Goudiaby, 2018a). Ceci fait des pratiques funéraires mayas un marqueur culturel très fort et ouvre à l'archéologie des voies peu habituelles. 
La fouille exhaustive des ensembles résidentiels mayas et l'observation soigneuse de la disposition des tombes au sein de ces espaces permet en effet d'identifier, avec une récurrence qui exclut le hasard, des individus qui se détachent du reste des défunts. De plus, cette esquisse de hiérarchie entre les morts de l'époque Classique fait écho à une autre plus récente, toujours en vigueur dans certains groupes mayas modernes. Du croisement entre archéologie et ethnologie émerge la figure de l'ancêtre : un mort différent des autres de par son influence et la déférence qu'il suscite chez les vivants, mais aussi un outil vecteur de pouvoir et, pour l'archéologue, une notion à manipuler avec précaution.

Nous nous proposons de montrer dans cette note comment l'archéologie funéraire peut identifier l'existence de ces distinctions subtiles entre défunts grâce à une étude contextuelle attentive. Puisque les sépultures mayas se fondent dans les espaces construits, notre postulat de départ consistera à superposer la hiérarchie des morts et celle des espaces domestiques, afin de mettre en évidence d'éventuels recoupements. Nous l'appliquerons à un exemple choisi tiré du site de Uaxactun (Guatemala), augmenté de quelques données ethnographiques. Nous verrons ainsi que certains morts sont plus proches des vivants que d'autres, et qu'il est possible de proposer un modèle de fonctionnement à même de fournir un cadre interprétatif valide.

\section{Principes de lecture spatiale}

À ce jour, on ne connaît toujours aucune aire funéraire dans la région maya (Webster, $2006: 6$ ). La notion de cimetière est virtuellement inexistante et le regroupement des morts ne semble pas dépasser les limites de la famille étendue. Ainsi, puisque les tombes se trouvent dans les habitats, la connaissance des principes propres à ces derniers peut nous guider dans l'étude des contextes funéraires associés. Deux en particulier nous intéressent plus spécifiquement. Le premier, et peut-être le plus important, est celui d'axialité. L'espace maya, public ou privé, est structuré autour d'axes dont les plus importants sont ceux qui passent par la porte centrale des bâtiments (Goudiaby, 2016 : 422). En effet, toute personne installée face à cette porte est la première que d'éventuels visiteurs verront : c'est donc une position prestigieuse. Lorsque les édifices sont disposés en carré, l'intersection de ces axes marque également un point central considéré comme particulièrement important. Notons que de façon générale, l'orientation des bâtiments mayas est presque toujours cardinale, et qu'il en va de même pour les sépultures.

Le second principe, difficile à énoncer de façon concise, est celui de la hiérarchisation par hauteur d'assise. Dans l'architecture maya, qu'elle soit périssable ou de pierre, des banquettes maçonnées sont souvent érigées dans les pièces. Hautes de 40 à $60 \mathrm{~cm}$, elles font à la fois office de surface de repos (lorsqu'elles se trouvent dans une zone privée, hors de vue) et de symbole hiérarchique (Tourtellot III, 1983 : 38-40 ; Lemonnier, 2006 : 7). On estime, sur la base des nombreuses représentations iconographiques qui vont en ce sens, qu'au moins dans les situations les plus formelles toute personne assise sur une banquette est plus importante que celles assises à son pied. Or, il se trouve que ces constituants marquants de l'espace maya abritent ou recouvrent souvent des sépultures. C'est donc un élément architectural susceptible de constituer à la fois un mobilier du quotidien, un signal hiérarchique et un marqueur funéraire.

\section{L'acropole A-V de Uaxactun}

Le site de Uaxactun se situe au nord du Guatemala, dans la région du Petén, à environ $20 \mathrm{~km}$ au nord du site plus connu de Tikal (tous deux connaissent une occupation synchrone durant l'époque Classique). Comme un certain nombre d'autres cités mayas, Uaxactun est pourvue d'une "acropole" : une construction massive dominant les alentours et supportant une série de bâtiments imbriqués (figure 1). Celle-ci est pourtant particulière dans la mesure où elle fut, avant d'être reconvertie en espace résidentiel de haut rang, un lieu qui abritait déjà les tombes de rois et autres dignitaires dans une série de temples funéraires (Smith, 1950). Ces premiers temples ont par la suite été engloutis dans une plateforme monumentale destinée à supporter les demeures des élites locales, construites sur deux niveaux, sans toutefois être entièrement détruits. On voit déjà, à ce stade, la concrétisation d'une volonté de la part des vivants de se lier à leurs défunts à travers une emphase mise sur la proximité physique. Ladite emphase va même plus loin, car non contents de s'installer directement au-dessus d'espaces funéraires symboliquement forts, les nouveaux occupants ont maintenu l'habitude d'inhumer certains de leurs morts dans les habitats. Cette superposition unique de deux mondes, entre lesquels la frontière est remarquablement ténue, a été exhaustivement documentée durant les années 1950 par les travaux d'Augustus L. Smith. Nombre de relevés, plans et tables détaillés, ainsi qu'un catalogue des inhumations dûment illustré ${ }^{1}$, donnent de l'acropole A-V une image remarquablement précise sur laquelle appuyer la réflexion. Notre discussion se concentrant sur les habitats, elle ne concernera que la phase résidentielle de l'occupation de l'acropole.

1 Quoique sans doute fidèles à la réalité du terrain en termes de disposition générale des restes, les relevés présentent nombre d'erreurs anatomiques et taphonomiques. Les proportions sont souvent erronées, de même que la forme de certains os. Cependant, dans un souci de respect du travail originel, les figures présentées dans cette note ont été redessinées par l'auteur directement à partir des relevés d'époque. Elles présentent donc les mêmes distorsions. 


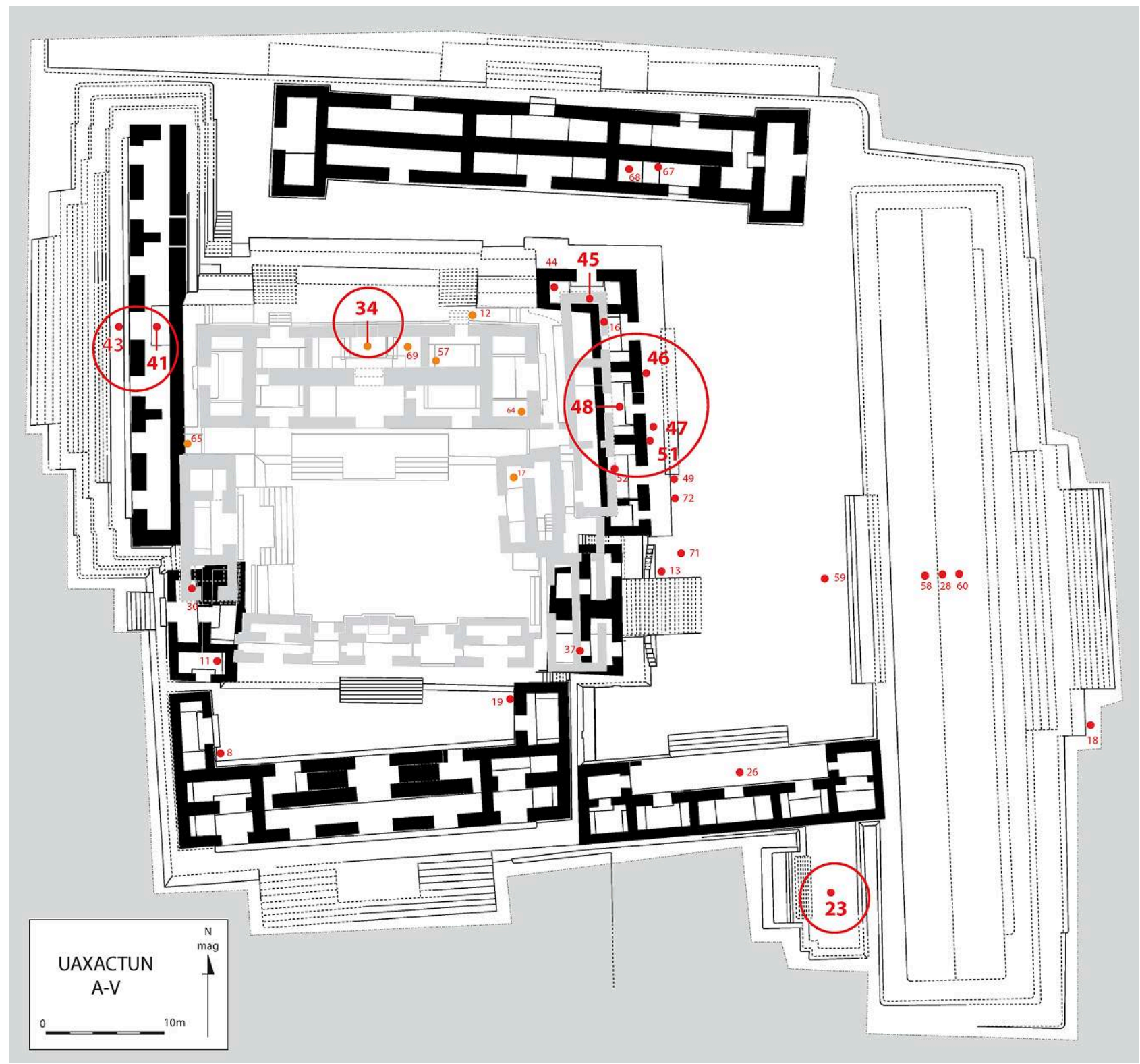

Figure 1. Plan de l'étape de construction finale de l'acropole A-V de Uaxactun indiquant la disposition des sépultures associées. Les bâtiments gris clair se situent au deuxième niveau / Map of the final phase of Uaxactun's Acropolis A-V, indicating the spatial distribution of associated burials. The buildings in light grey are on the upper level

\section{Les morts principaux}

L'application du principe d'axialité permet de mettre à part, sur les 58 sépultures que compte l'acropole de Uaxactun, un ensemble de 12 tombes, toutes mises en place au cours du Classique récent (600-950 apr. J.-C.). Au sein de ce sous-ensemble, on peut encore en isoler certaines qui semblent marquer des statuts différents entre les défunts. En effet, une sépulture située dans l'axe d'une pièce latérale n'a pas la même importance que celle qui se trouve dans l'axe du bâtiment entier, bien qu'elle soit plus prestigieuse que celles qui ne se trouvent sur aucun axe du tout. Suivant ce principe, 4 tombes se détachent tout particulièrement du lot. La plus marquante est certainement la sépulture 23 (figure 2), située à trois mètres de profondeur sous le centre même d'une plateforme qui a probablement été édifiée à la seule fin de l'accueillir (Smith, 1950 : 96). Le sujet, un adulte de sexe indéterminé, reposait en décubitus dorsal étendu, tête vers le nord. Il est très probable que cette sépulture ait été le point focal d'interactions directes entre les vivants et le défunt via des rites conduits sur la tombe de manière périodique ou cyclique. Des actions de ce type sont souvent mentionnées dans l'épigraphie et peuvent, dans certains cas, être poursuivies pendant plusieurs siècles (Eberl, 2005 ; Fitzsimmons, 2009).

Immédiatement inférieurs en termes d'importance et de visibilité, car installés dans des édifices préexistants (ce qui suppose un effort moindre), les occupants des sépultures 34 , 


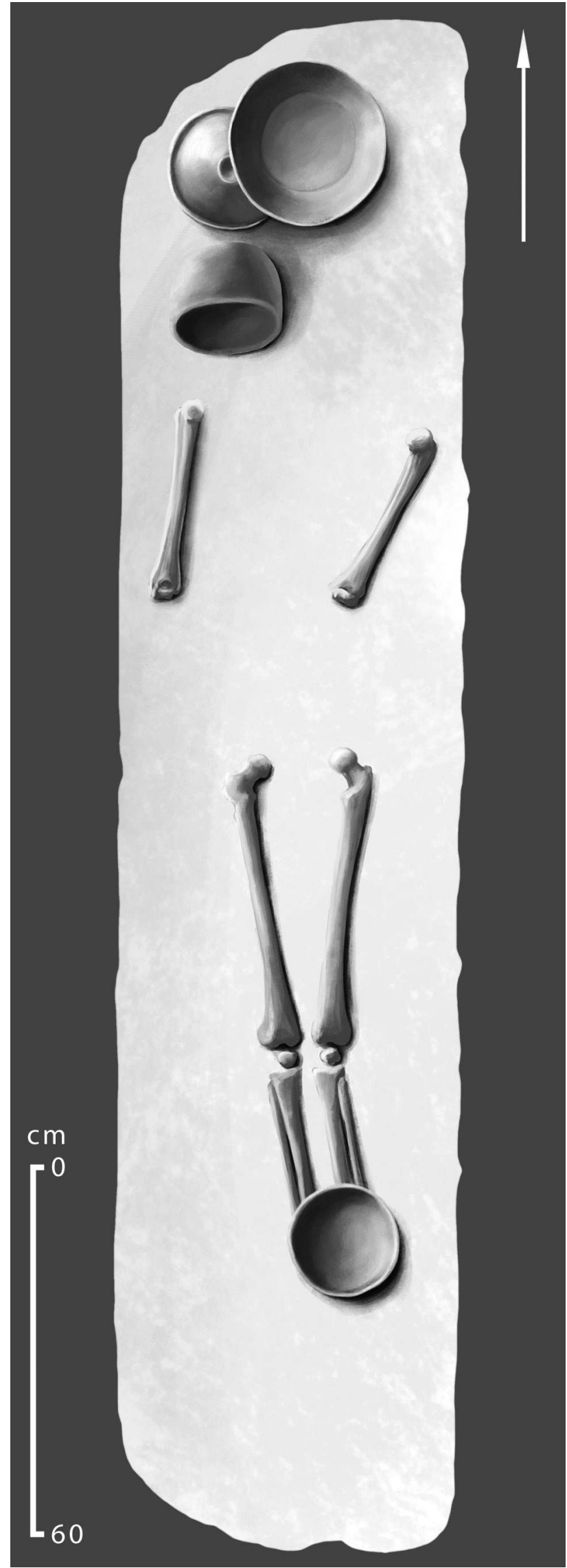

Figure 2. Relevé de la sépulture 23 (d'après Smith, 1950) / Drawing of Burial 23 (after Smith, 1950)
41 et 43 (Smith, 1950 : 98-99) étaient pourtant sans doute plus proches du monde des vivants que n'a pu l'être le sujet 23 . En effet, ils ont été placés dans des espaces de réception, tournés vers l'extérieur. 41 et 43 fonctionnent en tandem : la stratigraphie montre qu'elles ont été scellées par le même sol, et on remarquera qu'elles partagent le même axe. La sépulture 41, qui abritait une jeune adulte en décubitus dorsal étendu, est doublement marquée par son axialité et par une banquette qui se situait immédiatement à son aplomb. Ainsi, une personne assise sur la banquette se trouverait en hauteur, au centre d'un édifice à sept portes de plus de $20 \mathrm{~m}$ de long, associée à deux défunts dont l'emplacement évoque clairement un prestige certain. Quant à l'individu 34, un adulte masculin reposant lui aussi en position étendue, il est peut-être encore plus important que les deux précédents. La lecture du plan le place en effet au centre de l'acropole, en son point le plus élevé, sous une banquette à accoudoirs qui n'est pas sans rappeler l'allure d'un trône dans un référentiel occidental. Il ne fait guère de doute que cet individu jouissait d'un prestige considérable, lequel s'est non seulement préservé dans la mort mais rejaillissait après celle-ci sur ceux des vivants qui siégeaient directement sur sa sépulture.

\section{Les morts secondaires}

Le prestige des défunts mentionnés ci-dessus ne doit pas éclipser la présence d'autres qui, malgré des paramètres à première vue similaires (axialité, banquette...), se trouvent dans des espaces moins prestigieux ou de nature privée. Prenons l'exemple de l'individu 45 (figure 3) : il s'agit d'un adulte, probablement masculin, qui reposait en décubitus latéral droit dans l'axe d'une pièce ouvrant sur l'arrière de l'acropole (Smith, 1950 : 99). C'est là un espace nettement plus modeste et discret que les salles principales des grands édifices, et la position du sujet est également en rupture avec celles observées jusque-là. Pourtant, la personne a bel et bien été inhumée selon des critères similaires à ceux appliqués pour des individus prestigieux. Nous sommes donc probablement en présence d'un individu intermédiaire, important à l'échelle de sa maisonnée ou de son lignage, mais pas de la communauté tout entière comme c'était probablement le cas des précédents.

Un deuxième cas saillant parmi ces défunts "secondaires" est celui de l'occupant de la sépulture 48, car il s'agissait d'un sujet immature d'environ deux ans, déposé en décubitus dorsal étendu (Smith, 1950 : 99). Le contexte est presque identique à celui de la sépulture 45 : une banquette axiale dans une pièce annexe. L'âge du sujet ne laisse pas de surprendre dans ce contexte : trop jeune pour être considéré comme un "ancêtre" - au sens où il n'a pu engendrer de descendance -, probablement peu ou pas socialisé, l'hypothèse la plus probable est qu'il ait été inhumé non pas en fonction de ce qu'il était au moment de sa mort, mais d'un potentiel non réalisé. Sa position dans le lignage familial pouvait le promettre à un statut important qu'une mort prématurée l'aura empêché d'atteindre, auquel cas les vivants 


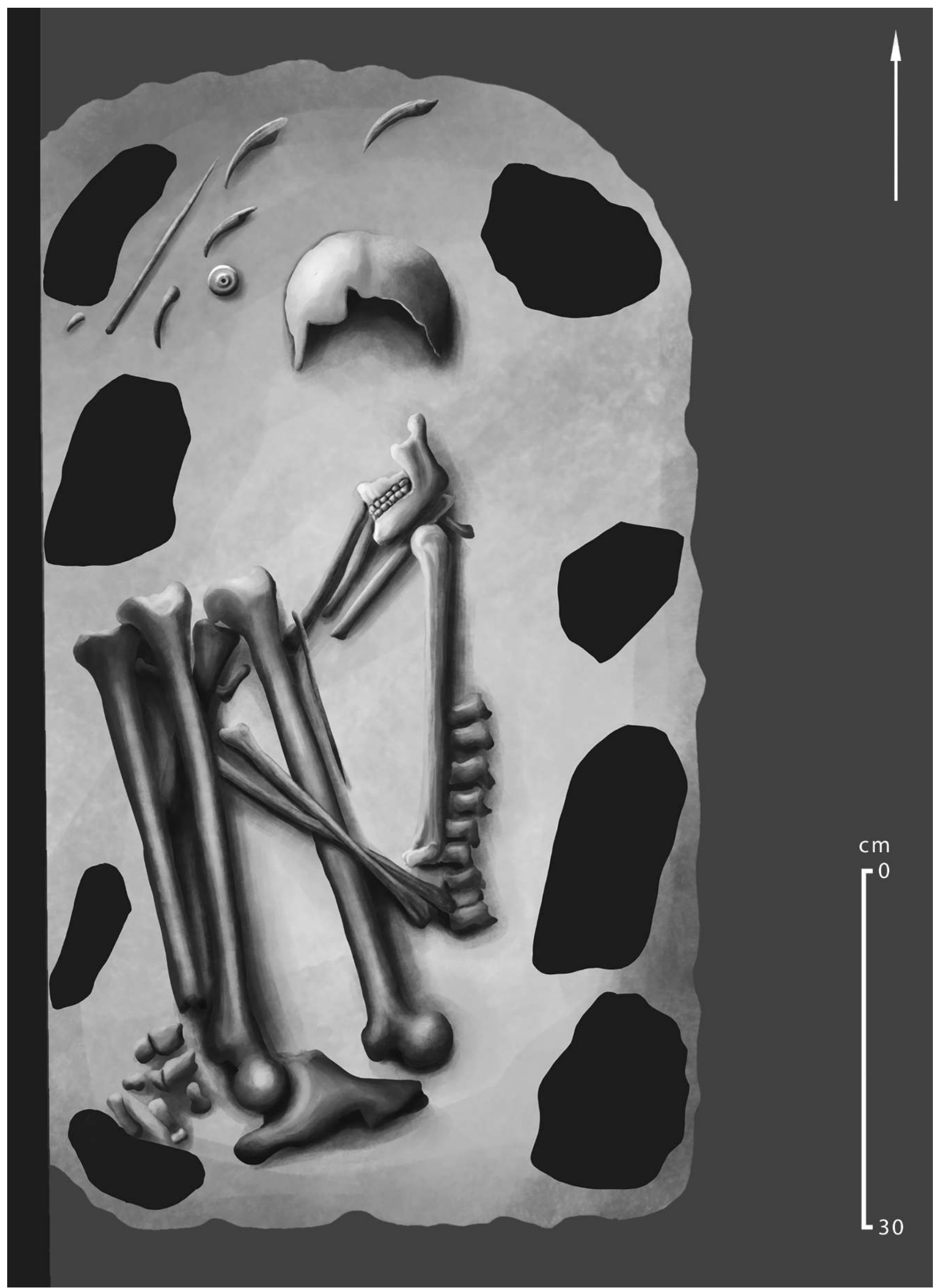

Figure 3. Relevé de la sépulture 45 (d'après Smith, 1950) / Drawing of Burial 45 (after Smith, 1950) 
l'auraient inhumé avec des honneurs bien supérieurs à son statut réel. C'est une explication très hypothétique, mais il faut souligner que les sépultures d'immatures sont relativement nombreuses dans l'acropole, et que nulle ne présente une telle conjonction de facteurs. Qu'il s'agisse d'une initiative isolée ou d'une mécanique d'intégration bien établie reste à déterminer. Cependant, à l'échelle de l'aire maya, l'apparition de jeunes immatures dans les assemblages funéraires est relativement peu fréquente (Uaxactun étant une exception à cet égard; Goudiaby, 2018a : 3, 17-18), et ils le sont plus encore dans des contextes de ce type. Nous pencherions donc plutôt pour la première option.

\section{Les morts dispersés}

Si l'on suppose que la localisation des tombes est bien inféodée au statut de leur occupant, il semble logique que la majorité d'entre elles se trouvent disséminées dans l'espace résidentiel, dans les bâtiments comme à l'extérieur. Elles appartiennent à des personnes d'importance moindre et obéissent donc à des codes différents. On remarque cependant que, de manière générale, les habitants de l'acropole semblent avoir évité de s'écarter trop des murs pour installer les défunts. D’autres cités comme Seibal se montrent moins embarrassées à l'idée de placer les morts un peu plus à l'écart, vers le centre des patios (Tourtellot III, 1988; Willey, 1990); c'est donc probablement une caractéristique locale.

Là où, dans le cas des sépultures axiales, il est assez aisé de nuancer l'interprétation sur la base du contexte, les sépultures que l'on qualifiera de "dispersées" sont plus difficiles à hiérarchiser car elles sont très homogènes. Les sépultures 46, 47 et 51 (Smith, 1950 : 99) illustrent bien la situation. Elles sont associées au même édifice que l'immature de la sépulture 48, et leur mise en place correspond à la même phase chronologique. Cependant, contrairement à lui, elles se situent le long de la façade extérieure. Très similaires entre elles, il s'agissait de fosses grossièrement délimitées par des pierres, abritant des sujets en décubitus latéral droit $(47,51)$ et gauche (46). Le sujet 46 était un adulte masculin, le 47 un adolescent de 15 à 18 ans (figure 4) enregistré comme masculin - mais la fiabilité de la détermination est ici douteuse - et le 51 une jeune adulte.

On notera, dans un premier temps, que ces individus plus âgés ont bénéficié d'un traitement moins prestigieux que l'individu 48 pourtant bien plus jeune. C'est à notre sens la manifestation claire d'une hiérarchie entre les morts qui impose de garder au plus près les plus importants en dépit de leur âge ou, probablement, de leur sexe. Cependant, il

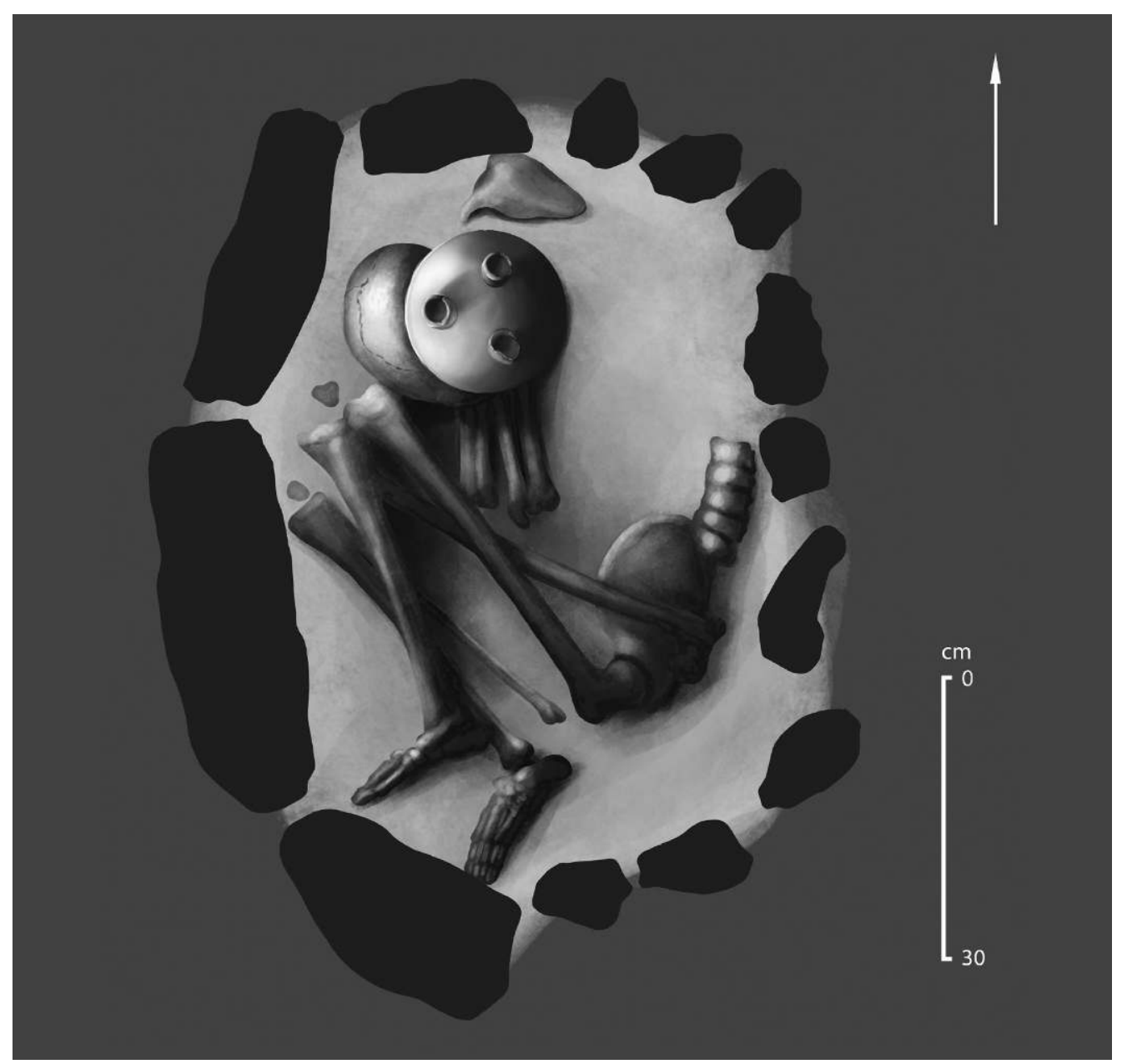

Figure 4. Relevé de la sépulture 47 (d'après Smith, 1950) / Drawing of Burial 47 (after Smith, 1950) 
est impossible de distinguer ces trois sépultures les unes des autres. Deuxièmement, le changement de position des squelettes est remarquable, car il ne se limite pas à ces trois individus. En effet, dès que l'on s'écarte des axes majeurs et des contextes importants, la norme de dépôt change elle aussi et les positions se font fléchies. C'est là une tendance que nous avons également pu observer sur le site voisin de Tikal (Goudiaby, 2016), cité suzeraine de Uaxactun. On peut donc avancer que le système funéraire de ces deux cités associe le décubitus dorsal étendu à un statut élevé. Cette observation n'est pas valide dans toute l'aire maya mais, dans le cadre de l'acropole de Uaxactun, elle est manifeste. Elle nous permet d'interroger non seulement les rapports entre les vivants et les défunts, mais aussi et surtout ceux des morts entre eux, car il est évident désormais que tous ne se valent pas. C'est ici que l'ethnographie peut apporter un éclairage particulièrement intéressant sur les données archéologiques.

\section{De l'apport de l'ethnographie}

La zone maya présente l'avantage considérable d'offrir d'une continuité culturelle qui autorise, pour peu que l'on prenne les précautions qui s'imposent dans de tels cas (Gallay, 2011 : 21-22), un certain degré de comparatisme entre les groupes mayas modernes et les contextes archéologiques. Rappelons donc, en premier lieu, l'importante acculturation et les altérations sociales provoquées par l'arrivée des Espagnols et la conversion subséquente au christianisme. En second lieu, les groupes mayas actuels présentent de grandes disparités en termes de croyances et de conception de la mort et des défunts. Au vu des variations observées d'un contexte funéraire et d'une cité à l'autre pour les périodes anciennes, tout porte à croire que cette variabilité existe de très longue date. Aucune observation, archéologique ou ethnographique, n'est donc directement transposable à l'autre discipline. Elles s'éclairent cependant mutuellement, comme nous allons maintenant l'illustrer.

\section{Genèse d'une hiérarchie}

Le cas des cérémonies mayas yucatèques actuelles, telles que décrites par Olivier Le Guen (2003, 2008), constitue un excellent point de repère théorique. En effet, ces rituels reconnaissent l'existence d'au moins deux grandes catégories de défunts, générées à l'issue d'un processus complexe et de longue durée. Au troisième jour après le décès, lors de l'inhumation - qui n'est donc pas immédiate -, "l'âme" du défunt (pixan) réalise son état de mort et entame son chemin vers l'au-delà, guidée en partie par les vivants (Le Guen, 2008 : 87). À compter de cet instant, des rituels sont entrepris tout au long de l'année à intervalles réguliers : tous les huit jours durant un mois, puis tous les trois mois jusqu'au premier anniversaire du décès. Le Guen interprète cette année de rituels cycliques comme une étape cruciale du processus d'ancestralisation de certains morts, et c'est là le point qui nous intéresse. Au terme de ces rituels, le défunt se voit intégré aux "ancêtres" familiaux ou aux collectifs, selon son importance dans la communauté.

Les morts dits "familiaux" ne sont révérés, invoqués ou évoqués que dans le cadre de la famille dont ils sont issus. Leurs sépultures se trouvent en général sur un terrain appartenant au lignage, voire même dans la cour centrale des habitats si les normes sanitaires l'autorisent. Les âmes, lorsqu'elles sont appelées lors de cérémonies spécifiques tenues au mois de novembre, viennent se restaurer auprès de l'autel familial. Elles le font alors selon un ordre bien défini : les âmes les plus proches de l'autel sont en général des morts identifiés, proches parents de l'officiant. Viennent ensuite des défunts plus éloignés dans l'arbre généalogique puis, derrière eux, une vaste catégorie de morts anonymes qui englobe "tous les autres". Remarquons que cette organisation des morts est un miroir exact de celle des vivants au moment du rituel : bien qu'à cette occasion la maison soit ouverte à tous, les plus proches de l'autel sont aussi les membres de la famille, puis la parentèle, et enfin d'éventuels visiteurs sans liens de sang.

Il existe également des morts dont l'importance, de par leur statut ou leurs accomplissements, dépasse celle du cadre familial. Ceux-ci seront plutôt inhumés en des lieux symboliques, comme un instituteur inhumé dans son école (Pacheco Cruz, 1947 : 27-28). La célébration des rites destinés à ces personnes notables se fait plutôt à l'église, où toute la communauté pourra participer. La séquence rituelle est elle aussi modifiée, puisqu'elle ne se limite plus cette fois à des groupes familiaux. C'est d'ailleurs aussi le cas lorsqu'une cérémonie concerne tous les morts, des plus connus à la masse des anonymes, sans distinction de rang. Ce dernier cas est très significatif. En effet, on appelle à cette occasion aussi bien des morts récents que d'autres tombés dans l'oubli de longue date, ce qui inclut ceux des sites archéologiques voisins. On voit donc ici la manière dont la division entre morts familiaux et collectifs se traduit spatialement et détermine le lieu d'inhumation, l'endroit où le rituel qui leur est dédié se tient, et la nature dudit rituel (Le Guen, 2008 : 103-104).

\section{Vers l'archéologie}

La projection directe d'un modèle aussi clair sur nos contextes archéologiques requiert d'autant plus de prudence qu'elle est tentante à première vue. Il convient de rappeler que, des trois critères mentionnés, seul le lieu d'inhumation est archéologiquement identifiable de façon certaine. Les deux autres ne le sont que dans des conditions excessivement rares et spécifiques. Deuxièmement, le cas que nous présentons ici ne solutionne pas la question du recrutement funéraire et des critères de sélection qui font qu'un mort sera retenu plutôt qu'un autre. Son apport principal réside dans la mise en évidence de hiérarchies imbriquées, rendant certains défunts plus influents et proches du monde des vivants que les autres, et se traduisant par des variations concrètes dans les pratiques funéraires. 
Dans le cas de Uaxactun et sur la base du seul critère spatial, on peut déjà supposer que les sujets qualifiés de "morts principaux" puissent correspondre à ces défunts définis comme "collectifs", qui maintiennent une forte identité dans l'au-delà grâce à une commémoration plus intense et à plus grande échelle. L'exemple le plus patent est celui des rois, auxquels la présence dans les textes glyphiques confère même la possibilité de sortir de l'oubli des siècles après leur disparition. On peut également penser que les morts inhumés sur des axes principaux, mais dans des espaces privés, correspondent aux défunts les plus importants à l'échelle de la famille : bien qu'ayant une place élevée au sein d'un lignage spécifique, leur rôle à l'échelle de la communauté n'est pas suffisant pour que leur influence post-mortem s'étende au-delà d'un groupe restreint. Quant à ceux qui sont inhumés à l'extérieur, il peut s'agir d'individus de rang encore inférieur que les vivants ont néanmoins voulu garder auprès d'eux. La masse des morts absents, qui ne sont pas présents dans les habitats, peut correspondre à "tous les autres": des défunts dont l'importance est reconnue en tant que groupe, mais dont l'individualité sera vite effacée de par leur intégration dans cette vaste catégorie. Les simulations démographiques effectuées en zone maya suggèrent que ces défunts inhumés hors de l'espace résidentiel représentent 90 à $95 \%$ de la population d'origine (Rice et Culbert, 1990 ; Haviland, 2003 ; Chase et Chase, 2004 ; Goudiaby, 2018b : 245-253). L'archéologie mayaniste interagit donc en fait avec des sous-groupes de défunts déjà soumis à une sélection particulièrement drastique : même ceux que nous avons qualifiés ci-dessus de "dispersés" sont en fait des individus privilégiés, de par leur simple intégration dans l'espace des vivants. Une question se pose alors : puisqu'ils forment un groupe à part, et malgré leur hiérarchie interne, les morts dans l'habitat sont-ils donc tous des ancêtres ? Quelle est la dynamique qui sous-tend ce système funéraire?

\section{Difficultés d'interprétation}

La variété des relations entre vivants et morts au sein des groupes actuels donne un aperçu du problème interprétatif posé par les contextes mayas. Les Chuj ne reconnaissent ainsi pour ancêtres, au sens de défunt identifiable et individualisé capable d'interventions dans la sphère des vivants (de Waal Malefijt, 1968 : 161-162), que des personnes ayant détenu une autorité religieuse (Petrich et al., 2009 : 257258). Ils sont d'ailleurs les seuls à conserver un nom et un prénom, afin de pouvoir être appelés après la mort. Les Tzeltal conçoivent leurs ancêtres en termes d'énergie qui peut se condenser dans un nouveau-né parmi ses descendants ; un ancêtre peut donc être à la fois mort et vivant, et la distinction entre les deux états n'est pas nette. Chez les Tzotzil, les interactions avec les totilme'iletik (pères-mères) font l'objet de rituels communautaires ; les K'iche, quant à eux, n'interpellent les morts que par grandes catégories (statut, profession, voire circonstances du décès ; cf. Breton, 1991). Ce bref aperçu montre qu'il serait déraisonnable, archéologiquement parlant, de mener l'interprétation audelà de la distinction familial/collectif évoquée plus haut. Du reste, celle-ci s'avère déjà très utile : sur cette base, on peut déjà proposer un schéma dynamique à partir duquel interpréter les contextes funéraires mayas (figure 5).

Parmi la population décédée, une immense majorité rejoindra immédiatement les "anonymes", individus dont l'identité s'effacera rapidement. Le terme n'a, en l'occurrence, rien de péjoratif. Une faible fraction, qui représente probablement les $10 \%$ visibles par l'archéologie, forme un groupe d'importance plus élevée. En son sein, les "aïeux"2 forment une catégorie d'importance intermédiaire, dont on peut envisager qu'elle comporte aussi bien des défunts individualisés que des groupes de défunts collectivisés selon des critères spécifiques. Toujours parmi les défunts spéciaux, une fraction encore plus réduite se voit véritablement élevée au rang "d'ancêtre", avec tout ce que cela suppose de pouvoir et d'influence non seulement pour le mort, mais aussi ceux de ses descendants qui peuvent en revendiquer protection et légitimité (McAnany, 2013). C'est parmi ces défunts que la distinction entre morts communautaires et familiaux devient essentielle, car ils sont les plus susceptibles de faire l'objet de séquences funéraires différentes des autres et d'être inhumés en des lieux symboliquement plus forts ${ }^{3}$. Enfin, l'introduction d'une marge d'oubli est elle aussi importante. Elle détermine à quelle vitesse le mort "meurt" à nouveau, socialement cette fois, pour rejoindre la masse des anonymes. Le "temps de l'oubli", individuel et collectif (Leclerc, 1990 : 16), constitue finalement la frontière la plus sûre entre vivants et morts, mais la vitesse à laquelle ces derniers la franchissent n'est pas la même. Les ancêtres sont ceux des morts qui, socialement, meurent le moins vite.

Pour boucler la boucle, et si l'on devait distribuer les sépultures présentées dans la première section de cette note parmi les catégories susmentionnées, le résultat serait probablement le suivant. Le sujet le plus important est sans doute le 23, qui correspondrait à un ancêtre communautaire individualisé. Les sujets 34, 41 et 43 semblent un cran en-deçà, mais ont tout de même été inhumés dans des espaces publics et prestigieux, en décubitus dorsal. Or, on l'a vu, position du corps et emplacement de la tombe semblent être deux paramètres cruciaux, contrairement au mobilier funéraire (peu abondant et remarquablement homogène en termes qualitatifs dans toutes les sépultures de l'acropole ${ }^{4}$,

2 Le terme "aïeul" a été retenu afin d'introduire une nuance entre ascendants familiaux directs, mais de statut moindre, et "ancêtres", dont le rôle et la présence sont plus actifs (entre autres au travers d'interventions directes).

3 Il faut également prendre en compte l'existence du sacrifice humain en zone maya, ainsi que d'éventuelles malemorts qui pourraient justifier une mise à l'écart de certains défunts.

4 Seules les chambres funéraires des élites les plus hautes, plus anciennes et mises en place alors que l'Acropole n'était pas encore un espace résidentiel, présentent une abondance de mobilier funéraire. Ainsi, ce critère de hiérarchisation perd rapidement sa pertinence au fur et à mesure que l'on descend l'échelle sociale. Voir Goudiaby, 2018a : 72-98 pour une discussion approfondie de ce critère en particulier. 


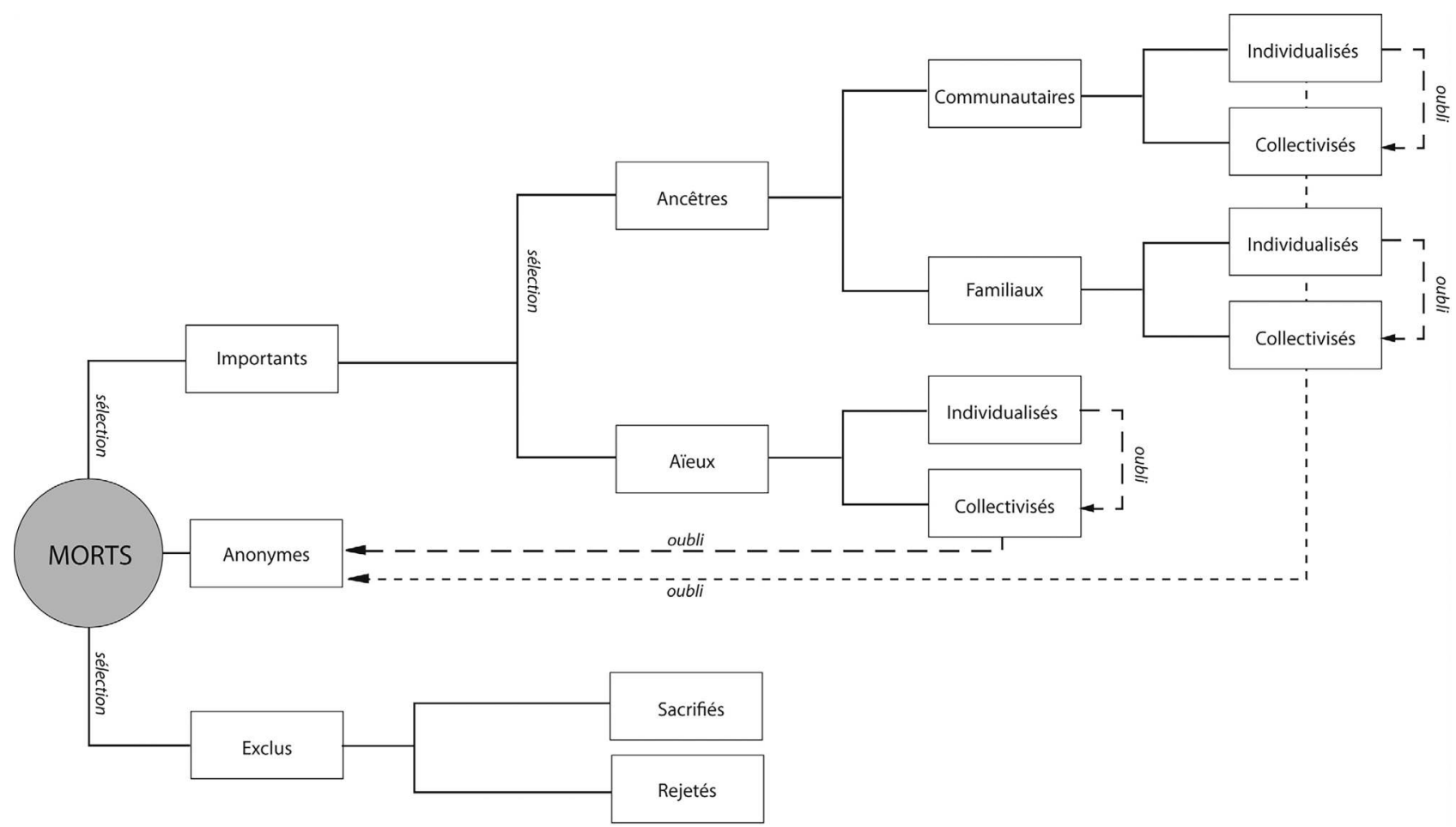

Figure 5. Proposition d'un modèle théorique des degrés d'ancestralisation dans les contextes funéraires mayas de la période Classique. Les morts sont distribués dans les différentes catégories en fonction de critères de sélection probablement liés au statut social des individus / Proposed theoretical model of the ancestralization process in Maya burials of the classic period. The dead were distributed into different categories that were probably linked to their social status

raison pour laquelle nous ne le développons pas ici ; $\mathrm{cf}$. Goudiaby, 2018a : 81). Ces trois sujets pourraient représenter des ancêtres communautaires collectivisés. Encore un degré plus bas, les sujets 45 et 48 , localisés sur des axes mais dans des zones privatives, correspondraient plutôt à des ancêtres familiaux. Enfin, les sujets 46, 47 et 51, placés légèrement à l'écart, pourraient être de simples aïeux.

\section{Considérations finales}

Le modèle que nous proposons n'a pas la prétention de refléter parfaitement une réalité générale ni d'être l'explication derrière la configuration des sépultures observée dans l'acropole A-V de Uaxactun, même si tout indique que notre postulat de départ (la mise en parallèle de la hiérarchie des morts et celle des espaces) est opérant. Nous le concevons plutôt comme un support de réflexion enrichi par l'ajout d'observations ethnographiques, qui apportent une profondeur appréciable à l'interprétation de ces contextes funéraires. Il est intéressant de chercher à déceler, au sein d'un échantillon qui a déjà fait l'objet d'une sélection, l'existence d'autres critères encore plus fins qui définissent des statuts différents au sein de ce sous-ensemble. Derrière l'apparente homogénéité suggérée par le terme "ancêtre", dont l'usage est trop souvent automatique (Sellato, 2002 : 2;
Whitley, 2002), se cache en fait un ensemble de morts "intermédiaires" qui, sans pouvoir prétendre à ce statut ultime, ne sont pas exclus d'un espace dont le recrutement s'avère pourtant hyper-sélectif.

Le cadre ethnographique montre par ailleurs que les interactions des vivants avec les morts sont souvent codifiées en fonction du statut de ces derniers. Il n'est pas simplement question de différences qualitatives en termes de dimensions des tombes ou d'abondance du mobilier. Le simple fait de maintenir une identité individuelle, un nom, est loin d'être acquis dans ces contextes d'habitat où les sépultures ne font l'objet d'aucun marquage et sont peu à peu recouvertes par les évolutions de l'architecture. Si la plupart de ces subtilités ne laissent pas de vestige matériel décelable par l'archéologie, l'analyse des pratiques et de la distribution des tombes dans l'espace en laisse entrevoir l'existence. En portant l'interprétation un peu plus loin qu'à l'accoutumée, et sans extrapoler au-delà de ce que les données autorisent, l'histoire individuelle et collective des habitants de ces espaces résidentiels se trouve considérablement enrichie. Ne pouvant traiter de vastes ensembles de centaines de sépultures, l'archéologie funéraire mayaniste livre une vision plus personnelle et intimiste du rapport entre vivants et morts au sein d'une société qui s'est ingéniée à en estomper la frontière. 


\section{Références}

Breton A (1991) Mayas : la passion des ancêtres, le désir de durer. Autrement, Paris, $285 \mathrm{p}$

Chase DZ, Chase AF (2004) Patrones de enterramiento y ciclos residenciales en Caracol, Belize. In: Cobos R (ed) Culto funerario en la sociedad maya: Memoria de la Cuarta Mesa Redonda de Palenque, $1^{\text {st }}$ edition. Instituto Nacional de Antropologia e Historia, México, D.F., pp 203-230

de Waal Malefijt A (1968) Religion and Culture: An Introduction to Anthropology of Religion. Waveland Press, Long Grove, $407 \mathrm{p}$

Eberl M (2005) Muerte, entierro y ascensión: ritos funerarios entre los antiguos mayas. Ediciones de la Universidad Autónoma de Yucatán, Mérida, 243 p

Fitzsimmons JL (2009) Death and the Classic Maya kings, $1^{\text {st }}$ ed. University of Texas Press, Austin, $321 \mathrm{p}$

Gallay A (2011) Pour une éthnoarcheologie théorique : mérites et limites de l'analogie ethnographique. Errance, Paris, 389 p

Goudiaby H (2016) Ancêtres et anonymes parmi les mayas : la norme de l'exception. In: Lauwers M, Zemmour A (ed) Qu'estce qu'une sépulture ? Humanités et systèmes funéraires de la préhistoire à nos jours. APDCA, Antibes, pp 413-429

Goudiaby H (2018a) Pratiques funéraires et statut des morts dans les ensembles résidentiels mayas classiques (250-950 apr. J.-C.) - volume II. Thèse de doctorat, Université Paris 1 Panthéon Sorbonne, $276 \mathrm{p}$

Goudiaby H (2018b) Pratiques funéraires et statut des morts dans les ensembles résidentiels mayas classiques (250-950 apr. J.-C.) - volume I. Thèse de doctorat, Université Paris 1 Panthéon Sorbonne, $345 \mathrm{p}$

Haviland WA (2003) Settlement, society, and demography at Tikal. In: Sabloff JA (ed) Tikal: Dynasties, Foreigners and Affairs of State. SAR Press, Santa Fe, pp 111-142

Le Guen O (2003) Quand les morts reviennent... Réflexion sur l'ancestralité chez les Mayas des Basses Terres. Journal de la société des américanistes 89:171-205

Le Guen O (2008) Ubèel pixan: el camino de las almas. Ancestros familiares y colectivos entre los mayas yucatecos. Península 3: $83-120$

Leclerc J (1990) La notion de sépulture. Bulletins et Mémoires de la Société d'anthropologie de Paris 2:13-1 [https://doi.org/ 10.3406/bmsap.1990.1738]
Lemonnier E (2006) La structure de l'habitat du site maya classique de la Joyanca (Petén Nord-Ouest, Guatemala) dans son environnement local. Archaeopress, Oxford, $259 \mathrm{p}$

McAnany PA (2013) Living with the Ancestors: Kinship and Kingship in Ancient Maya Society, Revised. Cambridge University Press, Cambridge, $258 \mathrm{p}$

Pacheco Cruz S (1947) Usos, costumbres, religión i supersticiones de los mayas: apuntes históricos con un estudio psicobiológico de la raza. E.G. Triay, Mérida, 152 p

Petrich P, Piedrasanta R, Figuerola H et al (2009) Variantes y variaciones en la percepción de los antepasados entre los mayas. In: Monod-Becquelin A, Breton A, Ruz MH (ed) Figuras mayas de la diversidad. UNAM, México, D.F., pp 255-275

Rice SD, Culbert TP (1990) Historical contexts for population reconstruction in the Maya lowlands. In: Culbert TP (ed) Precolumbian Population History in the Maya Lowlands. University of New Mexico Press, Albuquerque, pp 1-36

Sellato B (2002) Castrated dead: The making of un-ancestors among the Aoheng, and some considerations on Death and ancestors in Borneo. In: Chambert-Loir H, Reid A (ed) The Potent Dead: Ancestors, Saints, and Heroes in Contemporary Indonesia. Allen \& Unwin, London, pp 1-6

Smith AL (1950) Uaxactun, Guatemala : excavations of 1931-1937. Carnegie Institution of Washington, Washington DC, 108 p

Tourtellot III G (1983) An assessment of Classic Maya household composition. In: Vogt EZ, Leventhal RM (ed) Prehistoric Settlement Patterns: Essays in Honor of Gordon R. Willey. University of New Mexico Press, Albuquerque, pp 35-54

Tourtellot III G (1988) Excavations at Seibal, Department of Peten, Guatemala: peripheral survey and excavation, settlement and community patterns. Peabody Museum of Archaeology and Ethnology, Harvard University, $496 \mathrm{p}$

Webster D (2006) Introduction. In: Whittington SL, Reed DM (ed) Bones of the Maya. University of Alabama Press, Tuscaloosa, pp 3-12

Weiss-Krejci E (2006) Identifying ethnic affiliation in the Maya mortuary record. In: Sachse F (ed) Maya Ethnicity: The Construction of Ethnic Identity from Preclassic to Modern Times. Anton Saurwein, Markt Schwaben, pp 47-60

Whitley J (2002) Too many ancestors. Antiquity 76:119-126

Willey GR (1990) Excavations at Seibal, Department of Peten, Guatemala. Peabody Museum of Archaeology and Ethnology, Harvard University, $290 \mathrm{p}$ 